\title{
On the Conceptual Design of Waverider Forebody Geometries
}

\author{
Konstantinos Kontogiannis ${ }^{\dagger}$, András Sóbester ${ }^{\star}$ \\ University of Southampton, Southampton, UK \\ Nigel Taylor ${ }^{\S}$ \\ MBDA, Filton, $U K$
}

\begin{abstract}
This paper summarizes the results of investigations into the parametric geometry modeling of waverider forebodies, mostly centered on the osculating cones design method. Initially, three different approaches to controlling the leading edge shape are discussed. The first and most common method is driven by prescribing the upper surface trace on the base plane. The second incorporates a planform definition of the leading edge shape that gives more direct control of the sweep angle of the forebody. The third method directly controls the lower surface's trace on the base plane. The shockwave profile curve that defines the shape of the shock is defined by its trace on the base plane for all three cases. Each method provides direct control over different aspects of the geometry for which a desired shape would be more complex to obtain indirectly. We then estimate the level of flexibility required by the design-driving curves in the context of a design optimization study, in order to enable a variety of meaningful designs without needlessly complicating the geometry model. Additionally, we show an efficient and robust method to introduce bluntness to the leading edge of waverider forebodies utilizing rational Bézier curves.
\end{abstract}

\section{Nomenclature}

$\begin{array}{lll}\text { CFD } & =\text { Computational Fluid Dynamics } & \alpha=\text { local plane inclination angle } \\ \mathrm{L} / \mathrm{D} & =\text { lift over drag ratio } & \theta_{c}=\text { cone half angle } \\ \mathrm{LSPD} & =\text { Lower Surface Profile Definition } & \theta_{s}=\text { shockwave angle } \\ M_{\text {inf }} & =\text { freestream Mach number } & \\ \text { PLED } & =\text { Planform Leading Edge Definition } & \\ \text { RANS } & =\text { Reynolds averaged Navier-Stokes } & \\ \mathrm{R}_{\mathrm{c}} & =\text { radius of curvature } & \\ \mathrm{USPD} & =\text { Upper Surface Profile Definition } & \\ \mathrm{V} / \mathrm{A} & =\text { volumetric efficiency, volume/area } & \\ \mathrm{w}_{1} & =\text { rational Bézier curve weight } & \end{array}$

\section{Introduction}

$\mathrm{W}$

AVERIDER geometries are considered amongst the most promising in making hypersonic flight viable due to the high lift-to-drag ratios they exhibit at high speeds. This is accomplished by keeping the shockwave generated by the oncoming hypersonic flow attached to the entire leading edge of the lifting body, effectively trapping the high-pressure region of the shockwave at the underside of the body, greatly increasing the lift.

Since the introduction of the idea in the late $1950 \mathrm{~s}^{1}$, various approaches to designing such geometries have been proposed. Early inverse design methods were based on planar and axisymmetric shockwave flowfields, both of which were limited by the poor balance of L/D ratios, volumetric efficiency and ease of engine inlet integration. A more flexible design method that departed from the notion of having a single shock generating body is the osculating cones method ${ }^{2-3}$. The ability to shape the shockwave more freely enables significant improvements in the

\footnotetext{
${ }^{\dagger}$ Postgraduate Research Student, University of Southampton, Boldrewood Campus, Southampton, UK, SO16 7 QF.

* Associate Professor, University of Southampton, Boldrewood Campus, Southampton, UK, SO16 7QF; SMAIAA.

${ }^{\S}$ Capability Leader Aerodynamic Tools \& Methods, MBDA, PO Box 5, Filton, UK, BS34 7QW; SMAIAA.
} 
performance characteristics tradeoff, although it also results in a more complex design problem as the number of design variables increases. A further evolution of the method is the osculating flowfield method ${ }^{4}$, where the shock generating body for each plane is not restricted to a cone, allowing for greater flexibility in the design. As a number of key technologies advanced and the concept of an air-breathing hypersonic aircraft started to look increasingly feasible, later studies focused on more realistic designs, taking viscosity and thermal effects into account ${ }^{5-6}$.

A distinctive characteristic of hypersonic air-breathing aircraft designs is the degree to which the engine, inlet and nozzle must be integrated with the rest of the body, as their size relative to the aircraft increases in order to satisfy the needs of high-altitude hypersonic flight. The result of this is a very complex design problem that has to be tackled as a whole. It is therefore very important that the geometry parameterization is as efficient as possible, and to achieve that the waverider generation process can be slightly altered to better-fit specific design needs. The present work aims to provide more insight on handling parametric waverider geometries by first demonstrating three different implementations of the osculating cones method, each providing more direct control of a different aspect of the final geometry. Additionally, the amount of flexibility required by the method's design-driving curves is examined in order to ensure that the parameterization is capable of producing a variety of meaningful shapes without overcomplicating the geometry model.

Although the osculating cones method by itself is not enough to produce realistic hypersonic aircraft geometries, it provides a great tool for generating geometries with a leading edge attached shock, upon which more sophisticated designs can be based. One of the first issues that needs to be addressed when departing from the simplicity of inviscid designs is that of the aerodynamic heating effects that prohibit the use of sharp leading edges at hypersonic speeds. For that purpose an efficient and robust leading edge blunting approach is presented at the end of this paper. $\mathrm{MATLAB}^{7}$ was used for the majority of the computations presented.

\section{Osculating Cones Method}

The osculating cones method is an inverse design algorithm proposed by Sobieczky in $1990^{2}$. The method utilizes the axisymmetric flowfield of a shock-generating cone in supersonic flow, whose properties can be calculated by numerically integrating the Taylor-McColl equations. The design is driven by a curve controlling the shockwave shape, usually by defining its trace at the base plane (plane at the back of the geometry and perpendicular to the freestream direction), and another curve from which the shape of the leading edge on the shockwave can be determined. The flowfield solution is applied to osculating planes perpendicular to the local shockwave profile, while being scaled properly to match the curvature of the shock at each plane, as seen in Figure 1. The traces of the two design-driving curves (points 1 and 2) determine the leading edge trace on each plane (point 3). The lower surface of the waverider is then generated by tracing the streamlines of the flowfield on each plane from the leading edge to the base plane, from point 3 to 4 in Figure 2. As the stream-surface is replaced with a solid boundary, the flowfield downstream of the leading edge is replicated by the generated geometry alone. The upper surface is usually set to be parallel to the oncoming flow, although its shape can be manipulated both to further improve aerodynamic characteristics and create more space for the packaging of internal subsystems. The resulting flowfield is not an exact solution but a very good approximation, as small pressure gradients across different planes can cause some limited cross-flow. Applicability of the method has been experimentally validated ${ }^{8}$.

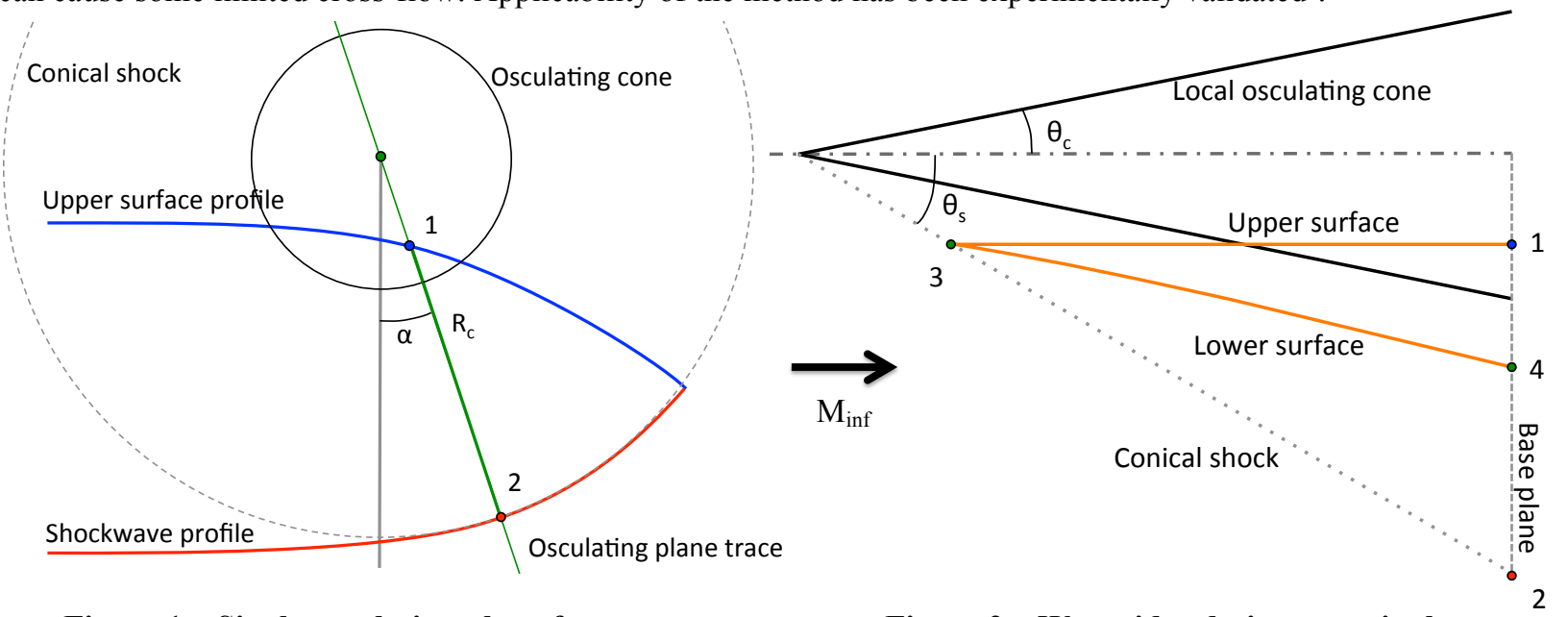

Figure 1 - Single osculating plane features on the base plane.

Figure 2 - Waverider design on a single osculating plane. 


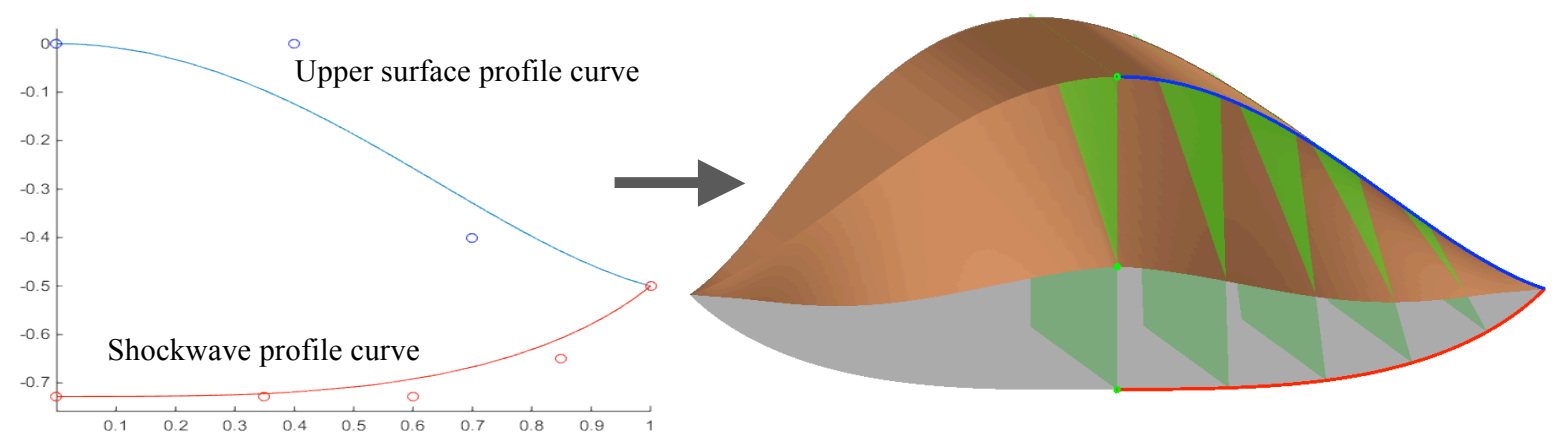

Figure 3 - Design-driving curves and resulting osculating cones waverider with the shock surface (grey), some of the osculating planes (green) and shockwave (red) and upper surface (blue) profile curves illustrated.

\section{Different Approaches to the Design Method}

The leading edge trace on the shockwave can be obtained in a number of different ways. Each one provides direct control of a different aspect of the geometry, something that can be exploited when there are specific design requirements or constraints to enable the geometry to more efficiently meet them. The shockwave shape itself is defined by a shockwave profile curve on the base plane in all cases.

\section{A. Upper Surface Profile Definition (USPD)}

The most commonly used approach is prescribing the upper surface profile on the base plane, along with the shockwave profile. The leading edge point on each osculating plane is then determined by moving upstream from the upper surface trace up to the shock, from point 1 to point 3 as illustrated in Figure 2. The implementation of this method is fairly straightforward as after picking an osculating plane and locating its point of intersection with the upper surface profile curve, the leading edge point can be trigonometrically located since the shock angle $\theta_{\mathrm{s}}$ is known. The limitation for the upper surface profile curve is that for each osculating plane, the trace of the upper surface profile curve on the plane must be between the shockwave profile trace and the center of the local osculating cone. If not, there is no feasible way to trace a streamline and the design process breaks down. Although having direct control over the shape of the upper surface is probably not very meaningful from an aircraft design standpoint, this method is very robust, utilizes two profile curves that are drawn on the same plane and gives good insight into the structure of the osculating plane-based geometry generation. An example of a waverider forebody generated with this method is presented in Figure 3.

\section{B. Planform Leading Edge Definition (PLED)}

The sweep angle of the lifting body's leading edge is a parameter that, depending on the design requirements, can be more efficiently controlled directly. The work of Rodi ${ }^{9}$ on geometrical relationships for osculating cones and osculating flowfield waveriders provides a set of useful mathematical relationships. They relate the angle between the upper surface profile curve of the previous approach and the shockwave profile, at their points of intersection on each osculating plane, with the sweep angle of the final geometry.

The method presented here differs in that the leading edge profile, as viewed from a planform view, can be designed directly. The upper surface profile curve at the base plane is, in this case, replaced by a leading edge profile curve on a horizontal plane. The leading edge point for each osculating plane is then located by finding the point of intersection between the shockwave trace and the vertical projection of the leading edge profile curve on the same plane, as seen in Figure 4. The lower surface is then generated by tracing the streamlines and the upper surface by moving in the freestream direction from that point to the base plane as usual, from 3 to 4 and 1 as seen in Figure 1.

The main constraint in this method is, similarly, the need for the leading edge point on each osculating plane to be behind the tip of the local osculating cone, otherwise stream tracing becomes infeasible. With this method, the shape of the leading edge remains identical to the leading edge profile curve from a planform point of view, but the leading edge's angle, which is a combination of the sweep and dihedral angles, can vary with respect to the freestream direction. Through Rodi's geometrical relations, the leading edge's angle relative to the freestream is instead directly controlled while the plan view sweep angle can vary. Both this and Rodi's approach can be 
appealing to use when facing specific design requirements. An example of a geometry generated with this method is illustrated in Figure 5.

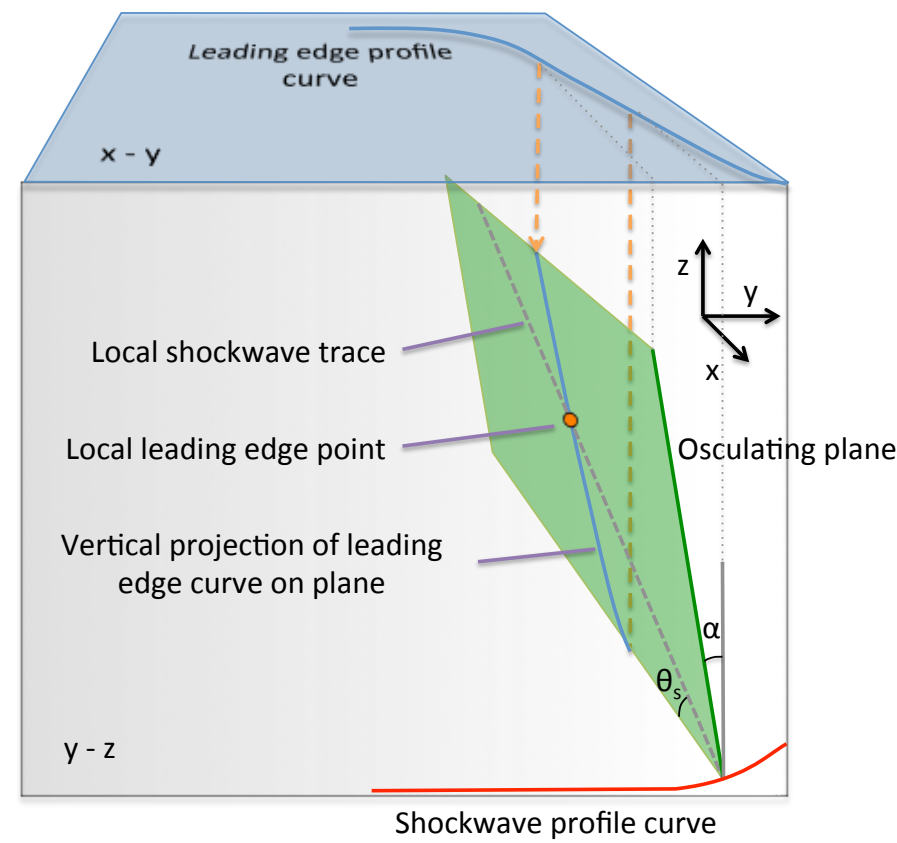

Figure 4-3D geometrical relations for locating the leading edge point on each osculating plane for a plan view definition of the leading edge shape.
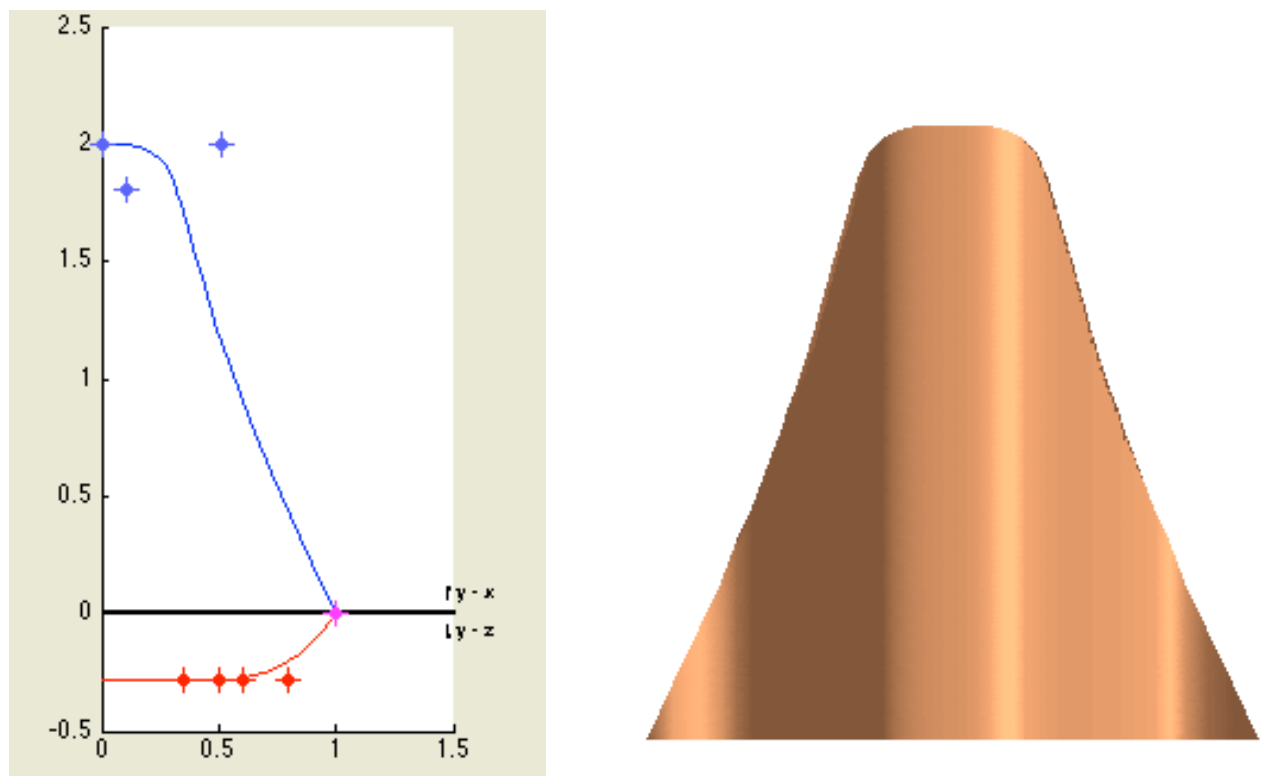

Figure 5 - The leading edge profile curve from a plan view (y-x plane), the shockwave profile curve on the base plane ( $y-z$ plane) and their control points (left), with the resulting waverider geometry (right).

\section{Lower Surface Profile Definition (LSPD)}

The third approach to the osculating cones method incorporates the direct definition of the lower surface trace at the base plane, and is essentially an inverse of the USPD method. A lower surface profile curve is used and its trace on each osculating plane is initially located. From that point, the lower surface is first constructed by tracing the 
streamline upstream up to the shock, which is where the leading edge point on the osculating plane lies, from point 4 to point 3 in Figure 1. The upper surface can then also be constructed by moving in the freestream direction from the leading edge point to the base plane.

The limitation in this method is that the trace of the lower surface profile on each plane must not be inside the local osculating cone, as that again would render stream tracing impossible. This approach gives more direct control of the lower surface shape at the base plane, where there might be specific needs for the engine inlet integration or a desired lower surface dihedral angle towards the tips of the lifting body. It is worth noting that as the relationship between the leading edge and the lower surface point at the base plane is realized though a stream tracing method of a flowfield that is scaled according to the local radius of curvature of the shockwave profile curve, the design can be very sensitive even to subtle changes. The geometry illustrated in Figure 6 is a good example of that, where for a simple flat lower surface profile at the base plane, the resulting geometry of the upper surface profile is much more complex.

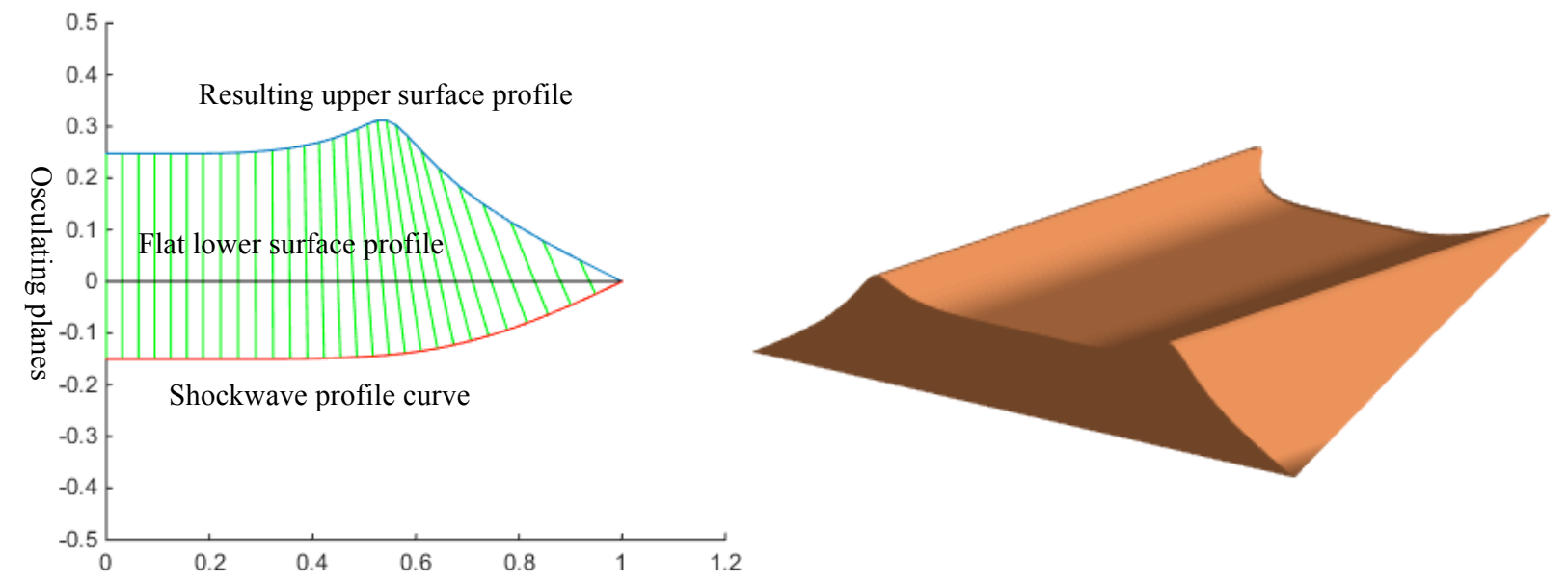

Figure 6 - The design's lower surface profile curve (black) and shockwave profile curve (red) with the resulting waverider geometry (right).

\section{Profile Curve Parameterization Options}

There are some strict limitations to the definition of the shockwave profile curve that are worth noting and are explained in Section A bellow. On the other hand, the curve describing the upper surface, leading edge or lower surface profile in each approach can be very flexible. As long as the limitations mentioned in Section III are not breached and the shapes are meaningful, any geometrical parameterization (such as splines or even line segments) can be used. This in turn leaves the designer with a great number of different options. Sections B and C provide insights into the effects of varying the amount of geometric flexibility made available for parameterizing simple waverider forebody designs.

\section{A. Shockwave profile curve}

As mentioned before, the design is very sensitive to the shape of the shockwave profile curve, mainly due to the fact that the local osculating cone flowfield on each plane is scaled according to the spanwise radius of curvature of the shockwave profile. Different scaling of the local flowfield affects both the curvature of the streamline and the location of the local shock-generating cone on each plane. Given a smooth leading edge, a smooth second order derivative (third order continuity) of the shockwave profile curve is needed in order to obtain a smooth lower surface. This is not an issue when a single infinitely differentiable function is used for the entire curve, but when piecewise functions are used, it needs to be considered. For example, a fourth order spline would be required to satisfy this condition.

In the material reported bellow, a line segment was used for the part of the shockwave profile under the center of the body where two-dimensional uniform flow that would favor the placement of an engine inlet is desirable. The rest of the shockwave profile curve was defined using a Bézier curve. In order to achieve third order continuity at the interface between the line segment and the Bézier curve, the first three intermediate control points of the curve have to be on a line. Merely making the shockwave profile curve smooth (first order continuity) with only the first point in line would result in a discontinuity of the resulting lower surface, while with a second order continuity the 
lower surface would simply not be smooth and an edge would be present at the resulting lower surface interface. Both cases illustrated in Figure 7.
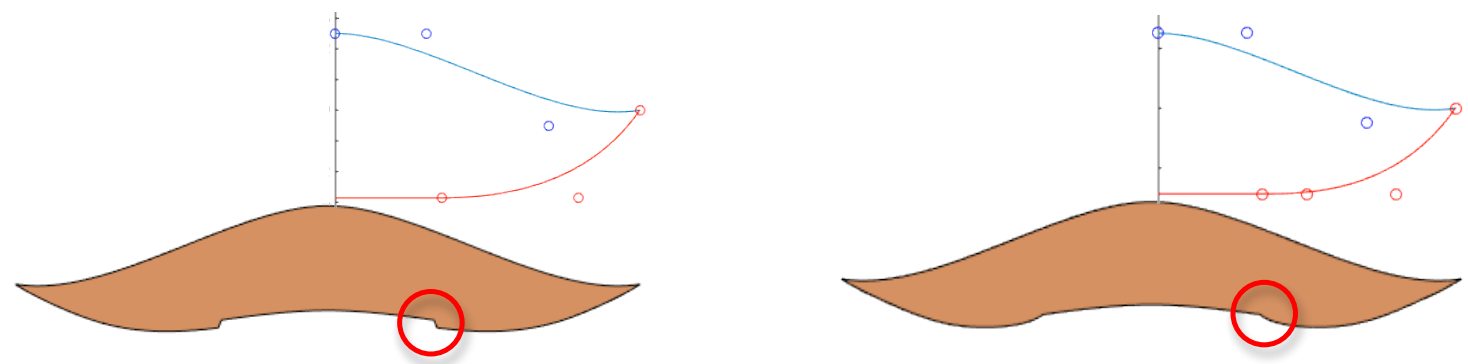

Figure 7 - First order (left) and second order (right) continuity at the interface between the straight line and the Bézier curve of the shockwave profile result in discontinuities and sharp edges in the lower surface.

\section{B. Matching geometries between different design methods}

While analyzing different parameterization options for the curve that controls the shape of the leading edge and to attempt to quantify the relative merits of the three approaches described above, we conducted a series of investigations where we attempted to 'reverse engineer' geometries by using a different method. Therefore we are, in a way, trying to answer the question of "how much control do we really need over this design-driving curve?" with "enough to be able to match geometries obtained using a different design method".

To replicate a geometry given by one of the design methods with another one, the design-driving curve has to match that geometry's respective shape. For example, a waverider forebody geometry given by the PLED method has a certain upper surface profile, which also depends on the shape of the shockwave. If for the same shockwave shape that upper surface profile is replicated by the parameterized curve of the USPD method, the same geometry can be obtained. The example presented here is the one mentioned in Section III-C, where a geometry with a flat lower surface profile is designed using the LSPD method. The resulting geometry has a more complex upper surface profile in contrast with the very simple line segment used for the driving curve, and although a peculiar case, serves the purpose of providing a clear comparison. To obtain a flat lower surface with the USPD method, the upper surface profile curve would need to match that upper surface shape of the LSPD generated geometry. To that goal, the best representations of that target upper surface shape using Bézier curves with increasing number of control points are presented in Figure 8.

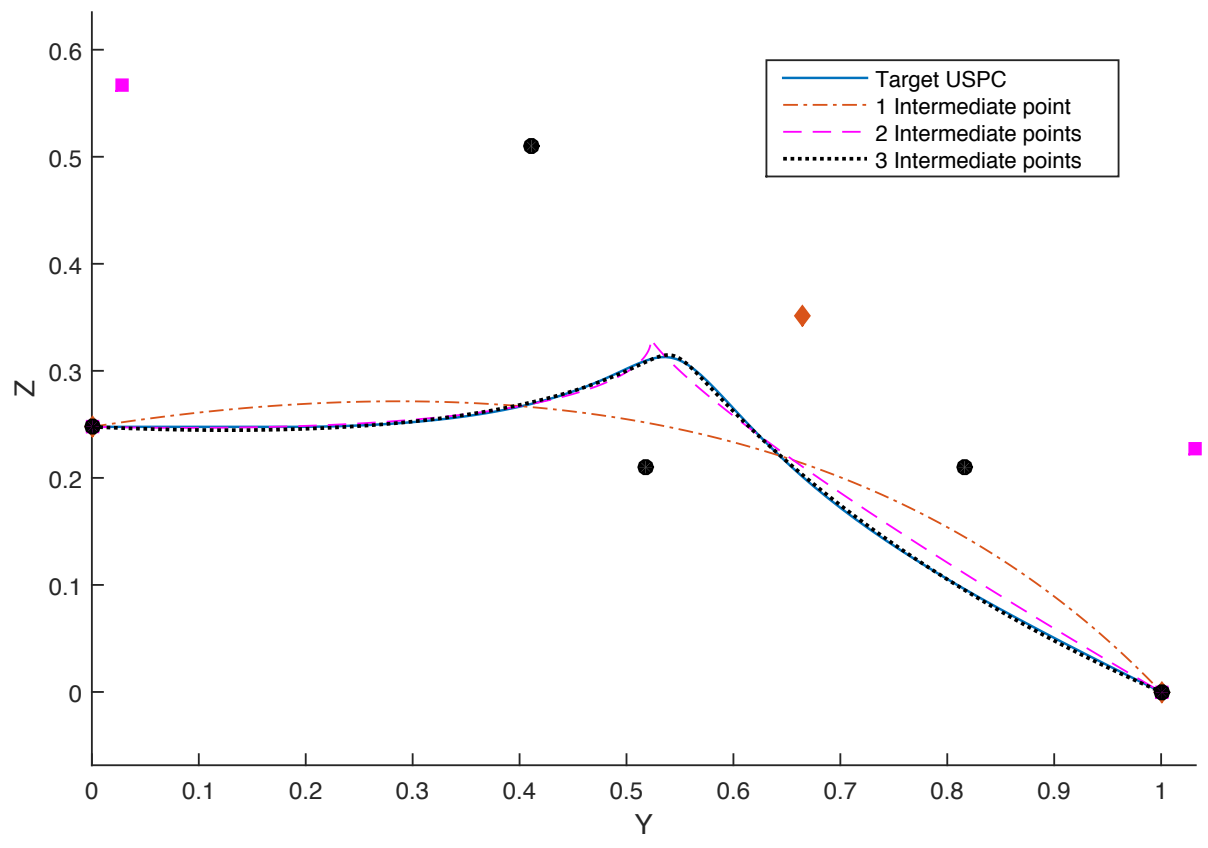

Figure 8 - Matching the upper surface profile of the flat lower surface design with 3, 4 and 5 control point Bézier curves. 
Using one intermediate control point is clearly not going to serve the purpose, but two or three points seem to improve approximation accuracy quite significantly. A curve using an additional fourth intermediate control point was also used and the deviations of each optimal representation from the target curve are shown in Figure 9, together with the resulting deviations of the resulting lower surface profiles of the forebody geometries. Figure 10 shows the final geometry using the two intermediate point approximation.
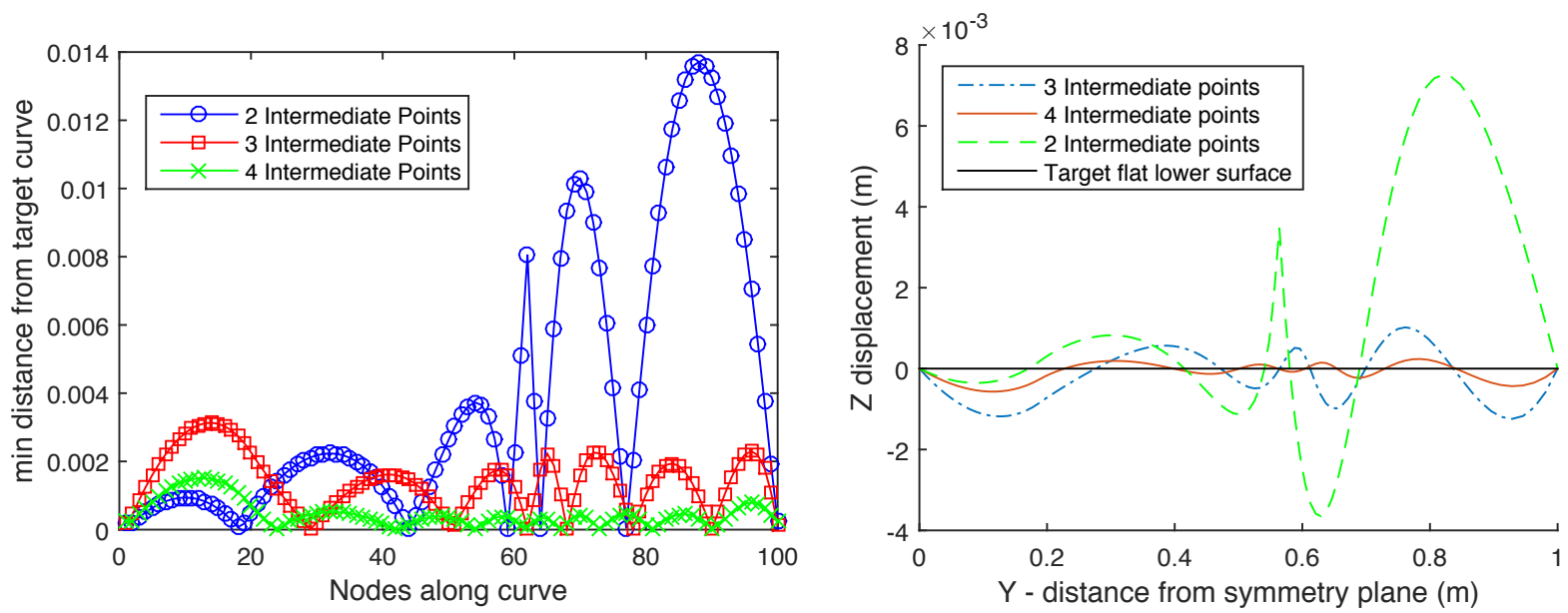

Figure 9 - Deviation of the parameterized curves from the target profile curve (left) and deviation of the resulting lower surface profile (right).
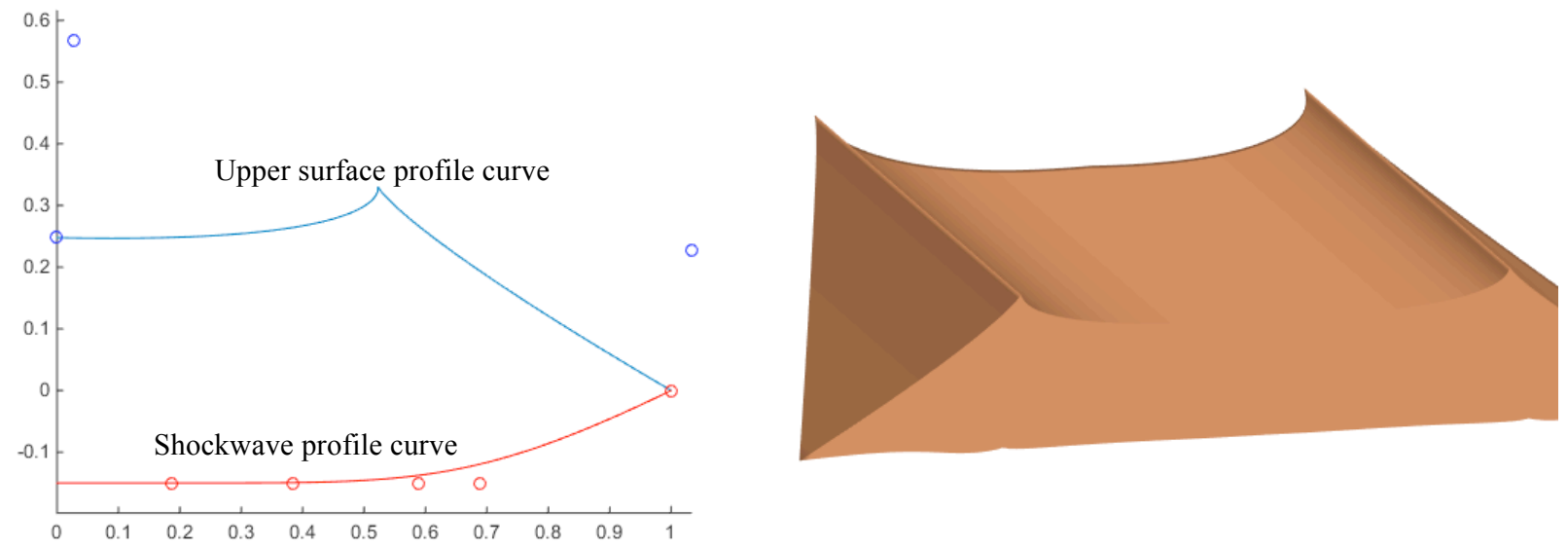

Figure 10 - Flat lower surface profile geometry approximation with the upper surface profile definition method and a third order Bézier curve ( 2 intermediate control points).

While in Figure 10 the imperfections are clearly visible in the final geometry, using one or two extra control points reduces them to the order of $\sim 1 \mathrm{~mm}$ for a geometry with a half-span of $1 \mathrm{~m}$. Therefore, to enable the geometry to achieve such shapes, at least two intermediate control points would be needed, i.e. four variables more than if the lower surface profile definition method was used. This result highlights the importance of method selection, especially in circumstances when design constraints or requirements can be related directly to the parameterization of a design-driving curve.

\section{Low-fidelity optimization studies}

Another approach to justifying geometry parameterization options before moving to higher fidelity and larger scale optimization studies of more realistic designs is to perform low fidelity optimizations in order to assess the parameterization itself. Aerodynamic performance characteristics of these waverider forebodies can be quickly estimated by using the flowfield values obtained by the Taylor-McColl equations for the lower surface and freestream values for the upper surface, with a boundary layer integral method to estimate viscous skin friction effects. A very simple method was implemented, assuming laminar flow for the entire forebody and with no interaction between the viscous and inviscid parts. Inviscid pressure forces and viscous skin friction forces are 
calculated for the wetted surface of the geometry (excluding the base - on which freestream static pressure was assumed to be acting throughout this analysis) and then integrated to obtain the resulting force on the body. As the two example comparisons presented in Table 1 show, the inviscid L/D results obtained in this way are within $\sim 0.5 \%$ of the results obtained from Euler CFD computations, while the viscous results are within $\sim 1.5 \%$ of laminar RANS computations. ANSYS Fluent ${ }^{10}$ was used for the comparisons. Since our objective is to develop the parametric geometry model, what matters most is relative changes of the characteristics between different designs and not the actual values themselves. In this context, this simplified approach for estimating aerodynamic performance is perfectly adequate. An investigation into the upper surface profile curve parameterization when using the USPD method will now be reviewed to illustrate the effects of increasing the complexity of geometric parameterization.

Designs were optimized for four different curve parameterizations using one, two and three intermediate control points (meaning three, four and five control point Bézier curves, respectively). The first three cases have one, two and three intermediate points with two degrees of freedom and therefore two design variables assigned to each of them. The fourth case has two intermediate control points equally spaced along the span and only able to move up and down. The shock shape is fixed as the current objective is confined to investigating the upper surface profile curve parameterization. A case for a Mach number of 8 and Reynolds number of $8.316 \times 10^{6}$ based on the length of the geometry is presented here. The two objectives to be maximized are the lift to drag ratio (L/D) and the volumetric efficiency (V/A), as these tend to be two of the main competing objectives for waverider designs. A simple genetic algorithm was used and a brute force approach taken given the low cost of the evaluations, so about 3000 designs were analysed for each parameterization. The resulting Pareto fronts and some of the geometries at their boundaries are presented in Figure 11.

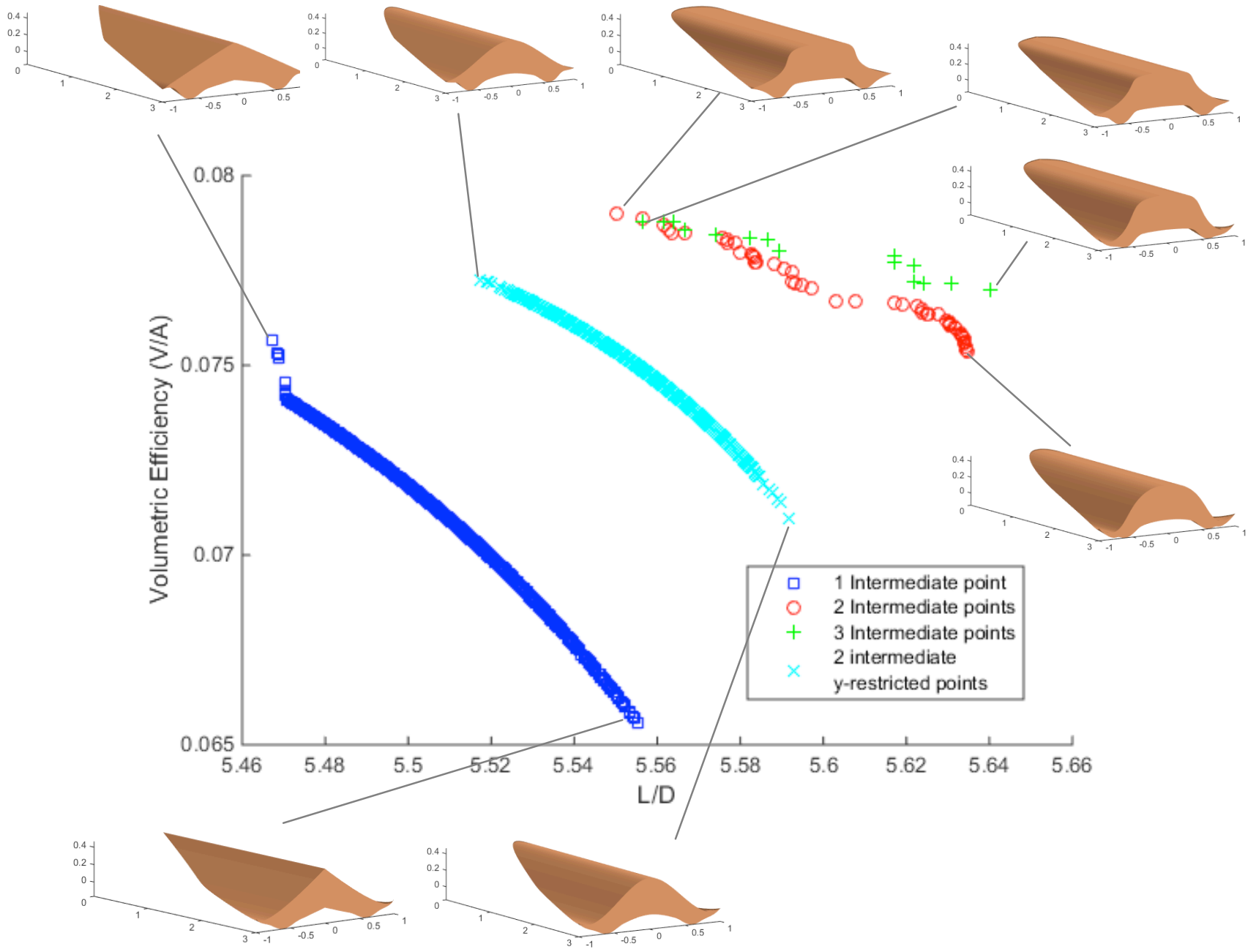

Figure 11 - Pareto fronts for different parameterizations of upper surface profile curve. 


\begin{tabular}{|c||c|c|c|c|}
\hline Geometry & Euler CFD & $\begin{array}{c}\text { Inviscid } \\
\text { calculated }\end{array}$ & $\begin{array}{c}\text { RANS laminar } \\
\text { CFD }\end{array}$ & $\begin{array}{c}\text { Viscous } \\
\text { calculated }\end{array}$ \\
\hline & 5.34 & 5.358 & 4.82 & 4.847 \\
\hline & 9.85 & 9.824 & 7.36 & 7.268 \\
\hline
\end{tabular}

Table 1 - Two of the inviscid and viscous $L / D$ calculation comparisons with CFD.

The Pareto fronts of the two design variable optimizations have about 900 designs each, the one with four design variables has 50 and the one with six only has 15 - a direct consequence of the increased number of design variables. The option of using a quadratic Bézier curve with a single intermediate control point seems to be the worst, as with the same number of design variables and using two intermediate control points with one degree of freedom, a much better set of optimal solutions can be obtained. Allowing the two intermediate control points to move in two directions yields a further improvement that would potentially be desirable should the increased cost of the optimization be acceptable. The same applies to using three intermediate control points, although the improvements are getting smaller while the cost of a design study with two more variables increases exponentially.

\section{Leading Edge Bluntness}

An efficient and robust approach to designing blunt leading edges for waverider forebodies has also been developed. The parameterization, illustrated in Figure 12, involves a quadratic rational Bézier curve whose middle point is placed in order to satisfy smoothness at the interface of both end-points, with the position of the two endpoints being determined by a truncation length of the original geometry. The shape of the curve can then be adjusted with the weight $\left(\mathrm{w}_{1}\right)$ of the middle point, while the truncation length controls the size of the blunted part. Therefore, compared to a circular arc shape (which can be described with a single design variable: its radius), by using an additional variable, the shape can also be controlled.
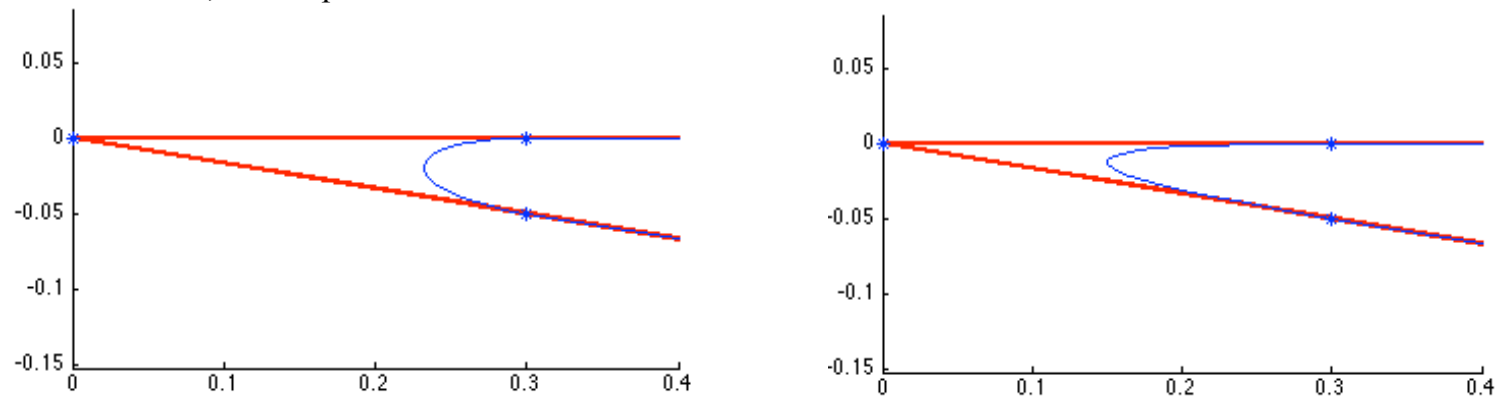

Figure 12 - Blunted leading edge (blue) with the rational Bezier control points compared to the sharp initial one (red) for values of $w_{1}=0.3$ (left) and $w_{1}=1$ (right).

In the work of Tincher et $a l^{6}$, it is hypothesized that instead of cutting material in order to introduce bluntness to the leading edge, it can be aerodynamically advantageous to raise the upper surface in order to create the necessary space to accommodate it. As a further addition to the parameterization the option to raise the upper surface can be incorporated as well, while both approaches to modifying the initial geometry can be blended providing a wider variety of geometries, as seen in Figure 13.

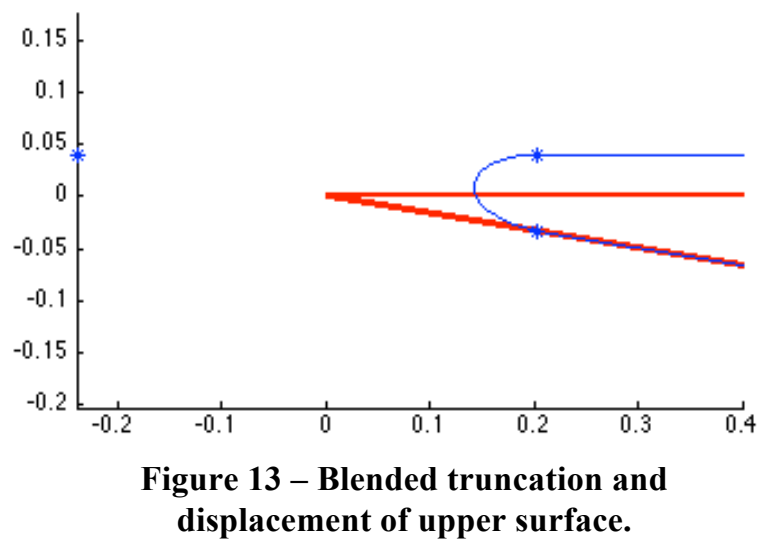


The parameterization is very robust and can provide a variety of meaningful shapes. The limited number of variables that control the shape make it well-suited for use in large-scale design optimization studies of full waverider geometries. The leading edge bluntness parameters can be set to vary between chosen values from the front to the wing tips, following a prescribed distribution or according to the sweep of the leading edge. More detailed CFD results will give more insight into the aerothermodynamic characteristics of the shapes.

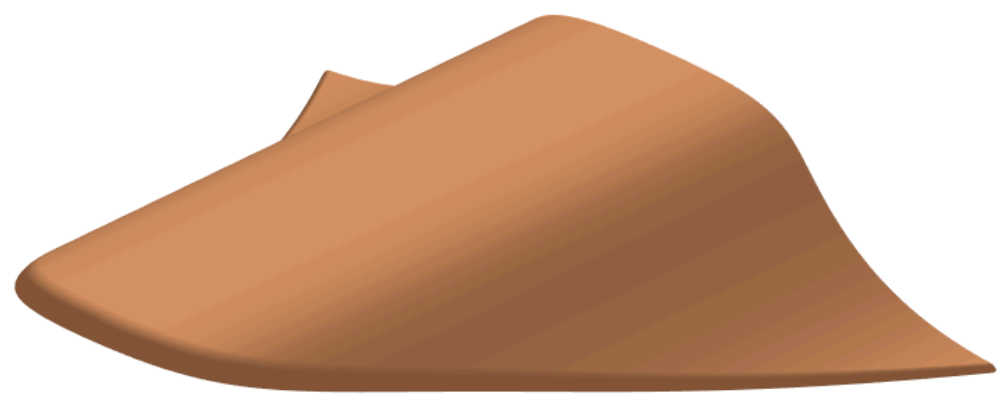

Figure 14 - Osculating cones waverider with varying bluntness parameters across leading edge.

\section{Conclusions}

In this paper the first stages of developing an efficient parametric geometry model for hypersonic aircraft have been presented. Three different applications of the osculating cones waverider forebody design method are documented and the importance of opting for the approach that is best suited to the design requirements has been highlighted. An analysis involving a series of multi-objective optimization studies was undertaken to determine the effect of increased flexibility of the design-driving curves on two performance characteristics of interest, with Bézier curves used mostly at this stage. Finally, an efficient and robust parameterization for introducing bluntness to the leading edge of waverider forebodies has been presented - although its characteristics are to be further investigated.

\section{Acknowledgments}

The authors would like to acknowledge the financial support provided by Innovate UK: the work reported herein has been undertaken as part of GHandI (TSB 101372), a UK Aerospace Technology Institute project.

\section{References}

${ }^{1}$ Eggers, A.J., Ashley, H., Springer, G.S., Bowles, J.V., and Ardema, M.D., "Hypersonic Waverider Configuration from the 1950's to the 1990's", AIAA Paper 93-0774, 31 st Aerospace Sciences Meeting, Reno, NV, 1993.

${ }^{2}$ Sobieczky, H., Dougherty, F., and Jones, K.D., "Hypersonic Waverider Design from Given Shock Waves", First International Hypersonic Waverider Symposium, University of Maryland, College Park, MD, 1990.

${ }^{3}$ Center, K.B., Sobieczky, H. and Dougherty, F.C., "Interactive Design of Hypersonic Waverider Geometries", AIAA Paper 91-1697, AIAA 22 ${ }^{\text {nd }}$ Fluid Dynamics, Plasma Dynamics and Lasers Conference, Honolulu, HI, June 1991.

${ }^{4}$ Rodi, P.E., "The Osculating Flowfield Method of Waverider Geometry Generation", AIAA Paper 2005-0511, 44 AIAA Aerospace Sciences Meeting, Reno, NV, 2005.

${ }^{5}$ Bowcutt, K.G., Anderson, J.D., Capriotti, D., "Viscous Optimized Hypersonic Waveriders", AIAA Paper 87-0272, $24^{\text {th }}$ Aerospace Sciences Meeting, Reno, NV, 1987.

${ }^{6}$ Tincher, D., Burnett, D., "Hypersonic Waverider Test Vehicle: A Logical Next Step", Journal of Spacecraft and Rockets, 31 , 392-399, 1994.

${ }^{7}$ MATLAB Release 2014b, The MathWorks, Inc., Natick, Massachusetts, United States.

${ }^{8}$ Mill, R.W., Argrow, B.M., Center, K.B., Brauckmann, G.J., and Rhode, M.N., "Experimental Verification of the Osculating Cones Method for Two Waverider Forebodies At Mach 4 and 6," AIAA Paper 98-0682, 36th Aerospace Sciences Meeting, Reno, NV, 1998.

${ }^{9}$ Rodi, P.E., "Geometrical Relationships for Osculating Cones and Osculating Flowfield Waveriders", AIAA Paper 20111188, 49 ${ }^{\text {th }}$ Aerospace Sciences Meeting, Orlando, FL, 2011.

${ }^{10}$ ANSYS FLUENT, Release 14.0, ANSYS, Inc. 